\title{
A One-year Observational Study on Hand Hygiene Practices in an Open Intensive Care Unit of a Large Teaching Hospital in India
}

\author{
${ }^{1} \mathrm{P}$ Naveen Kumar, ${ }^{2}$ Shivakumar, ${ }^{3} \mathrm{Uttham}$ Sharma
}

\begin{abstract}
Health care-associated infection (HCAl) is the commonest complication affecting hospitalized patients. The infection sources in a hospital are personnel, hospital's inanimate environment, and self-infection. Direct transmission accounts to $70 \%$ of infection transfer. Bacteria, fungi, and viruses have been reported as causative agents in HCAls, and many infections are polymicrobial. Effective safety management in the 21st century involves keeping an eye on human factors and highly reliable organizations can identify and capture potential hazards before they show themselves as accidents. One method of achieving this is by measuring the level of safety through "leading" indicators. An active surveillance to measure the adherence to hygiene of hands of employees is conducted by the infection control nurses randomly in the critical areas in this hospital. Hand Hygiene Moment Observation Form taken from the original World Health Organization (WHO) 5 Moments "Observation Form" is used for observation. The compliance among nurses is greater compared with doctors in the intensive care unit (ICU), 64.44 to $60.74 \%$. During the said period, the number of infections incidence in ICU compared with overall hospital's infections was $62.98 \%$. The nurses had 7 opportunities and doctors had 10 opportunities to wash hands during 1-hour period, and the average time spent for washing was 8 seconds by doctors and 14 seconds by nurses.
\end{abstract}

Keywords: Hand hygiene of health care workers, Health careassociated infections, Infection control nurse, Patient safety, World Health Organization observation form study.

How to cite this article: Kumar PN, Shivakumar, Sharma U. A One-year Observational Study on Hand Hygiene Practices in an Open Intensive Care Unit of a Large Teaching Hospital in India. Int J Res Foundation Hosp Healthc Adm 2018;6(1):17-21.

\section{Source of support: Nil}

Conflict of interest: None

\footnotetext{
${ }^{1}$ Associate Professor, ${ }^{2,3}$ Junior Resident

${ }^{1-3}$ Department of Hospital Administration, Kasturba Medical College \& Hospital, Manipal Academy of Higher Education Manipal, Karnataka, India
}

Corresponding Author: P Naveen Kumar, Associate Professor Department of Hospital Administration, Kasturba Medical College \& Hospital, Manipal Academy of Higher Education Manipal, Karnataka, India, Phone: +919019164899, e-mail: naveenpdr@yahoo.co.in

\section{INTRODUCTION}

Modern medicine has been thorough, wonderful, and genuine, but modern medicine too has the potential for considerable harm, perhaps even greater harm than in the past. ${ }^{1}$ The health sector when compared with other sectors is a high-risk area as the adverse events arising from treatment rather than disease can lead to death, complications, and further sufferings. Though many hospitals have procedures in place to ensure patient safety, the health care sector lags behind other industries and services that have introduced systematic safety processes, famous being airline industry and health care industry comparison in all scientific gatherings and presentations.

Patient safety can be defined as: "The avoidance, prevention and amelioration of adverse outcomes or injuries stemming from the process of health care," as per Vincent. ${ }^{1}$

Health sector-induced harm to patients imposes a heavy burden on society. Investment in patient safety, therefore, has the potential to generate savings in expenditure coupled with an obvious benefit to patients. If a hospital focuses on patient safety, it leads to savings in treating patients manifesting adverse events and subsequent improved use of financial resources of the community as such. Supplementing this, savings are achieved in litigation costs associated with grievances and applications for compensation related to adverse events. Of utmost important, patient safety bestows better quality of life. In order to achieve these measures, the culture of safety can be improved significantly in various ways.

It is said that we cannot manage what we cannot measure. The purpose of evaluating patient safety is that it helps to effectively monitor safety and quality or assess the impact of any initiatives or programs that are implemented. The systematic reporting and tracking of safety problems is an important approach to quality improvement.

Nosocomial infection, or HCAI, is the commonest complication affecting hospitalized patients. Currently, 5 to $10 \%$ of patients admitted to hospital in Britain and in the US acquire one or more infections; millions of people each year are affected. ${ }^{1}$ In a massive survey of over 75,000 patients in 2006 , a prevalence rate of $7.59 \%$ 
in the UK was found. ${ }^{2}$ The sources of infection are personnel, hospital's inanimate environment, and selfinfection. Bacteria, fungi, and viruses have been reported as causative agents in HCAIs, and many infections are polymicrobial. The most common causative agents are Staphylococcusaureus, Pseudomonasaeruginosa, Escherichia coli, Enterococci, Candidaalbicans, and Klebsiellaspecies. ${ }^{3}$ In the wards, hospital infections may manifest in the form of bacteremia, respiratory infection, gastroenteritis, urinary tract infection (UTI), meningitis, and skin infections. Surgical wound infections are found after surgery. Four types account for about $80 \%$ of nosocomial infections: UTIs often associated with catheter use; bloodstream infections, often due to intravascular devices, surgical site infection (SSI), and pneumonia. Each of these four types may arise in more than one way and may be due to one or more different bacterial species. Intravenous lines are a particular potent source of infection, and if the chance of infection is increased, the longer the line remains in place. ${ }^{1}$ Nowadays, infection control covers a wide range of processes within a hospital. It needs epidemiological expertise and attention to medical devices (e.g., intravascular and enteral devices, ventilators, diagnostic equipment); the physical environment (e.g., ventilation ducts, physical surfaces); surgical wound management; and carrying by health care employees and supportive health professionals. ${ }^{4}$

Diabetic patients, dialysis patients of chronic renal failure, and with chronic dermatitis are most likely to have areas of intact skin that are colonized with Grampositive bacteria. ${ }^{5-12}$ Skin flakes containing microorganisms are shed daily from normal skin and are 100 in number approximately. ${ }^{13}$ The patient gowns and fomites in the patient's immediate environment can be easily contaminated with patient flora. ${ }^{14,15}$ During patient care activities like lifting a patient, taking patient's pulse reading, oral temperatures, and blood pressure, which involves patient touching, can contaminate nurses' hands with 100 to 1,000 colony forming units of Klebsiella spp. ${ }^{16}$

In health care situation, the health care providers if called as "donors" and patients as "recipients," the transmission of organisms from donor fabrics to clean recipient fabrics through hand contact has also been studied. The results explicably showed the number of organisms transmitted was greater if the donor fabric or the hands were wet during contact process. ${ }^{17}$

Hospital-acquired pneumonia (HAP) is one of the most common infections occurring in patients getting care in an ICU and accounts for almost $25 \%$ of all nosocomial infections in ICU patients, with incidence rates ranging from $6 \%$ up to $52 \%$. Prevention and control of HAP include: Staff education and their involvement in preventing infections, prevention of infection transmission; sterilization or disinfection and maintenance of equipment, devices, mechanical ventilators; prevention of person-to-person transmission of organisms; standard precautions, such as hand hygiene, glove, and gown usage; care of patients with tracheostomy; suctioning of respiratory tract secretions. ${ }^{18}$

Few steps in the prevention of central line-associated blood stream infections (CLABSI) include education, training and staffing, appropriate selection of catheters and sites, hand hygiene and aseptic techniques, maximal sterile barrier precautions, following catheter site dressing regimens, etc. that comply with current Centers for Disease Control and Prevention (CDC) hand hygiene guidelines. ${ }^{19}$ Effective patient safety management in the 21st century includes paying attention to human factors, physical factors, and environmental factors. By paying attention to human factors, highly reliable organizations can identify and capture potential hazards before they exhibit as accidents. One method of achieving this is by measuring the state of safety through indicators, such as safety culture or climate. These are seen as different from indicators of safety, such as incidents as they provide knowledge into the state of safety without the need for retrospective analyses. ${ }^{20}$ World Health Organization provides a set of quality indicators to be captured; most of the indicators are aimed at patient safety. We studied the indicators that have a bearing on hand hygiene of the health care personnel that would lead to increased infection rates.

\section{MATERIALS AND METHODS}

An observational study was conducted for a period of 1 year, January 2016 to December 2016, in the multidisciplinary ICU of this tertiary care teaching hospital. This is an Open ICU with admission and discharges done by respective specialty consultants. The tool used for observation was WHO 5 Hand Moments Observation tool. Informed consent was taken from the doctors and nurses prior to the start of study, and repeated once every quarter. All personnel entering this Open ICU were included in the study.

\section{RESULTS AND DISCUSSION}

The study was carried out in multidisciplinary 24-bed ICU at a tertiary care teaching hospital. The hospital has emerged as a premier health care institution providing quality tertiary care to patients. It was started as a 150bedded teaching hospital in 1961. It has grown since its inception to 2,032-bedded superspecialty tertiary care teaching hospital. The hospital received 608,788 outpatient and 73,308 inpatient admissions in a year. The hospital is 
committed to patient safety. The hospital follows patient safety practices through various measures undertaken. This study relates to hospital-acquired infection, safety, and sentinel events. Infection control is an important component of patient safety having direct bearing on the prevention of HCAIs and the transmission of infectious agents among patients and health care providers. Infection control manual is prepared and periodically reviewed by the Hospital Infection Control Committee, at this hospital. The infection control policies include:

- Hand hygiene policy.

- Standard precautions for health care workers.

- Guidelines for hospital waste management, and sharps disposal policy.

- Antibiotic policy.

\section{Hand Hygiene Policy}

The hospital hand hygiene policy promotes hand hygiene and provides a comprehensive indication for hand washing, which include before touching a patient, before a procedure, after a body fluid exposure risk, after touching a patient, and after touching a patient's surroundings. Hand hygiene policy is followed as per WHO guidelines. ${ }^{21}$

\section{Definition}

Hand washing is the vigorous rubbing together of lathered hands for at least 15 to 20 seconds, followed by thorough rinsing under a stream of clean water.

\section{Purpose}

- To remove transient microbial contamination that has been acquired by recent contact with infected or colonized patients or environmental sources.

- To prevent the transmission of potentially pathogenic organisms.

The policy provides standard duration of hand hygiene practice:

- Two minutes at the beginning of duty.

- Two minutes before high-risk procedures: Intravenous handling, catheter care.

- Two minutes after heavy contamination: Dressing changes.

- Fifteen to 30 seconds for other routine hand washing with alcohol hand rub.

- Six-minute scrubs are required for preoperative hand washing.

The products for hand washing include soap solution, hand rub solution (alcohol-based), and products containing antiseptic preparations: Topical antimicrobials are used to disable, inactivate, or kill microorganism and reduce total skin bacterial count.
Hand washing facilities are provided in all the patient care areas. Hand rub solution is provided at each patient bed in critical areas like ICUs, postoperative wards, at the entrance of each ward, and at all nursing counters.

Techniques for hand hygiene depends on: ${ }^{22,23}$

- Amount of solution

- Duration of washing procedure

- Selection of hand hygiene agents

- Alcohol-based hand rubs are the best agents for reducing the number of bacteria on the hands; antiseptic soaps and detergents are the next most effective; and nonantimicrobial soaps are the least effective.

- Water and soap are recommended for clearly soiled hands.

- Alcohol-based rubs are recommended for decontamination of hands for all patient-related situations (except when hands are visibly soiled) and as one of the options for surgical hygiene.

An active surveillance to measure the adherence to hand hygiene is conducted by the infection control nurses randomly in the critical areas. Hand Hygiene Moment Observation Form adapted from the original WHO 5 Moments "Observation Form" is used for observation (Annexure 1). The infection control nurse observes the doctors, nursing staff, paramedical staff, and student nurses for adherence to Hand Hygiene. The 5 Moments for Hand Hygiene are:

1. Before touching a patient.

2. Before clean/aseptic procedure.

3. After body fluid exposure risk.

4. After touching a patient.

5. After touching patient surroundings.

Compliance rate is measured using the formula $\rightarrow$ Compliance $(\%)=\frac{\text { Actions }}{\text { Opportunities } \times 100} \rightarrow$

The highest hand hygiene compliance was seen among student nurses $(71.81 \%)$ and paramedical staff (70.94\%), followed by nursing staff (64.44\%) and the least was among doctors (60.74\%). The compliance among nurses is greater compared with doctors. This has been observed in other studies also. The compliance rate in the national hand hygiene audit conducted in Australia showed compliance rates of $64.6 \%$ among doctors, $82.4 \%$ among nurses, 77\% among paramedical staff, and 76.9\% student nurse. ${ }^{24}$ In a similar study the hospital's compliance rate was $50.3 \%$, and its distribution among doctors was $49.1 \%$, nurses $52.2 \%$, and technicians $42.8 \%$. $^{25}$

During the same period, the number of HCAIs observed in the hospital was 543, out of which 342 (62.98\%) were observed in ICUs and 201 (37.02\%) outside the ICUs. The total patient days was 529,718 days in a year. The overall HCAI rate was 1.025 per 1,000 patient days 
or 0.74 per 100 admissions $(0.74 \%)$. The HCAI rate in the ICUs was $1.98 \%$ as compared with $0.36 \%$ in the wards. The observed HCAI rate is below that estimated by CDC. The CDC estimated the burden of US HCAIs in 2002 as 1.7 million infections in hospitals (9.3 infections per 1,000 patient days or 4.5 per 100 admissions). ${ }^{26}$ The WHO study shows a hospital-wide prevalence of HCAI varies from 5.7 to $19.1 \%$, with pooled prevalence of $10.1 \%{ }^{27}$

The nurses had seven opportunities to wash their hands during 1 hour in ICU, and an average period the washing was performed was 12 seconds. Whereas, the doctors had 10 opportunities to wash their hands during 1 hour, and an average period the washing performed was 8 seconds. The duration of hand washing or hygienic hand wash episodes by health care workers has averaged 6.6 to 24.0 seconds in various observational studies.

The most common site of HCAI was HAP $47.7 \%$, out of which ventilator-associated pneumonia $(21.18 \%$ of HCAI) accounts for $44.4 \%$ of HAP. The second most common infection was catheter-associated UTI (24.49\%), followed by SSI (16.21\%), infected burns wound (6.26\%), and CLABSI (5.34\%).

In the USA, the most frequent type of infection hospital-wide is UTI (36\%), followed by SSI (20\%), bloodstream infection, and pneumonia (both $11 \%$ ). ${ }^{28}$ A study in European acute care hospitals reports pneumonia (37.3\%) to be the most common HCAI site followed by BSI (18.6\%), SSI (11.9\%), and UTI (3.4\%). ${ }^{29}$

The most common organism causing HCAI overall was Acinetobacter species (19.19\%) followed by E. coli $(13.18 \%), P$. aeruginosa (13.18\%), and $K$. pneumoniae $(12.79 \%)$. Methicillin-resistant $S$. aureus was seen in $4.65 \%$ of HCAI. The study in European acute care hospitals reports the most common causative organism was Enterobacteriaceae (34\%), out of which E. coli was the most common. Candida species were the second most frequently detected pathogens (17\%), followed by Enterococci $(15 \%)$, S. aureusisolates $(6 \%)$, and $P$. aeruginosa isolates $(6 \%){ }^{29}$

Responsibilities of patient care managers/administrators are:

- Statements can be displayed about the value and adherence to hand hygiene practices.

- Role models demonstrating adherence to recommended hand hygiene practices. ${ }^{30}$

\section{Glove}

Gloves should be worn while handlings blood/infected body fluids, handling of items on surfaces soiled with blood or body fluids, for all invasive procedures, venipuncture, and other vascular procedures. Gloves should be changed between patient-to-patient contact. Gloves should be used while handling visibly soiled linen. Wearing gloves does not replace the need for hand hygiene. ${ }^{31}$ After providing care for a patient if gloves are not changed, it may lead to transmission of microorganisms from one patient to the other. ${ }^{32}$

By November 2017, this hospital got certified by Guinness Book of Records for the hand hygiene awareness drive, for clearing a world record in highest number of congregation of hand washing done in a day's time. The Department of Hospital Administration and we, as members of the department of the hospital, overlooked the conducting of event. The hospital has started promoting physicians and surgeons as brand ambassadors of hand hygiene in the premises of the hospital. The hoardings are displayed in the outpatient area, operation theatre complex, and intensive care areas.

\section{CONCLUSION}

This study relates the proportion of hospital-acquired infections with hand hygiene practices by health care professionals in a hospital setting. Infection control is an important component of patient safety concerned with the prevention of HCAIs and the transmission of infectious agents among patients and health care workers. Among the published literature search on reasons for poor adherence with hand hygiene recommendations, all are clearly associated either with institution or system or people. Visible safety programs across the hospital should be the norm of quality policy. An awareness program among workers, encouraging workers to report, a tolerant and supportive attitude toward reported problems, and belief in the efficacy will reap better dividends for hospitals in the long run.

\section{REFERENCES}

1. Vincent, C. Patient safety. 2nd ed. West Sussex: WileyBlackwell Publishing Ltd; 2010.

2. Smyth ET, McIlvenny G, Enstone JE, Emmerson AM, Humphreys H, Fitzpatrick F, Davies E, Newcombe RG, Spencer RC; Hospital Infection Society Prevalence Survey Steering Group. Four country healthcare associated infection prevalence survey 2006: overview of the results. J Hosp Infect 2008 Jul;69(3):230-248.

3. Jarvis, WR. Bennett \& Brachman's hospital infections. 5th ed. Philadelphia (PA): Lippincott Williams \& Wilkins; 2007.

4. Institute of Medicine. To err is human: building a safer health care system. Washington (DC): National Academy Press; 1999.

5. Tuazon CU, Perez A, Kishaba T, Sheagren JN. Staphylococcus aureus among insulin-injecting diabetic patients. JAMA 1975 Mar;231(12):1272.

6. Kaplowitz LG, Comstock JA, Landwehr DM, Dalton HP, Mayhall CG. Prospective study of microbial colonization of the nose and skin and infection of the vascular access site 
in hemodialysis patients. J Clin Microbiol 1988 Jul;26(7): 1257-1262.

7. Aly R, Maibach HI, Shinefield HR. Microbial flora of atopic dermatitis. Arch Dermatol 1977 Jun;113(6):780-782.

8. Kirmani N, Tuazon CU, Murray HW, Parrish AE, Sheagren JN. Staphylococcus aureus carriage rate of patients receiving long-term hemodialysis. Arch Intern Med 1978 Nov;138(11): 1657-1659.

9. Goldblum SE, Ulrich JA, Goldman RS, Reed WP. Nasal and cutaneous flora among hemodialysis patients and personnel: quantitative and qualitative characterization and patterns of staphylococcal carriage. Am J Kidney Dis 1982 Sep;2(2):281-286.

10. Boelaert JR, Van Landuyt HW, Gordts BZ, De Baere YA, Messer SA, Herwaldt LA. Nasal and cutaneous carriage of Staphylococcus aureus in hemodialysis patients: the effect of nasal mupirocin. Infect Control Hosp Epidemiol 1996 Dec;17(12):809-811.

11. Zimakoff J, Pedersen FB, Bergen L, Baagø-Nielsen J, Daldorph $B$, Espersen F, Gahrn Hansen B, Høiby N, Jepsen OB, Joffe P, et al. Staphylococcus aureus carriage and infections among patients in four haemo- and peritoneal-dialysis centres in Denmark. J Hosp Infect 1996 Aug;33(4):289-300.

12. Bibel DJ, Greenbert JH, Cook JL. Staphylococcus aureus and the microbial ecology of atopic dermatitis. Can J Microbiol 1997 Aug;23(8):1062-1068.

13. Noble WC. Dispersal of skin microorganisms. Br J Dermatol 1975 Oct;93(4):477-485.

14. Bonten MJ, Hayden MK, Nathan C, VanVoorhis J, Matushek M, Slaughter S, Rice T, Weinstein RA. Epidemiology of colonisation of patients and environment with Vancomycin resistant enterococci. Lancet 1996 Dec;348(9042):1615-1619.

15. Walter CW, Kundsin RB, Shilkret MA, Day MM. Spread of staphylococci to the environment. Antibiot Annu 1958-1959;6:952-957.

16. Casewell M, Phillips I. Hands as route of transmission for Klebsiella species. Br Med J 1977 Nov;2(6098):1315-1317.

17. Marples RR, Towers AG. A laboratory model for the investigation of contact transfer of micro-organisms. J Hyg (Lond) 1979 Apr;82(2):237-248.

18. Ranes, JL.; Gordon, S.; Arroliga, AC. Hospital-acquired, health care-associated, and ventilator-associated pneumonia. Cleveland (OH): The Cleveland Clinic Foundation; 2000-2011.

19. Edmond MB, Wallace SE, McClish DK, Pfaller MA, Jones RN, Wenzel RP. Nosocomial bloodstream infections in United States hospitals: a three-year analysis. Clin Infect Dis 1999 Aug;29(2):239-244.
20. Yule, S. Senior management influence on safety performance in the UK and US energy sectors. Doctoral thesis, University of Aberdeen, Scotland. 2003.

21. WHO. Clean care is safer care. Geneva: WHO; 2015. [cited 2017 Mar 30]. Available from: http://www.who.int/gpsc/en/.

22. Rotter, M. Hand washing and hand disinfection (Chapter 87). In: Mayhall CG, editor. Hospital epidemiology and infection control. 2nd ed. Philadelphia (PA): Lippincott Williams \& Wilkins; 1999.

23. Boyce, JM. Scientific basis for handwashing with alcohol and other waterless antiseptic agents. In: Rutala WA, editor. Disinfection, sterilization and antisepsis: principles and practices in healthcare facilities. Washington (DC): Association for Professionals in Infection Control and Epidemiology, Inc; 2001.

24. National Hand Hygiene Compliance Rate-Australia National Data Period Two, 201.

25. Bukhari SZ, Hussain WM, Banjar A, Almaimani WH, Karima TM, Fatani MI. Hand hygiene compliance rate among healthcare professionals. Saudi Med J 2011 May;32(5):515-519.

26. Klevens RM, Edwards JR, Richards CL Jr, Horan TC, Gaynes RP, Pollock DA, Cardo DM. Estimating health care-associated infections and deaths in U.S. hospitals, 2002. Public Health Rep 2007 Mar-Apr;122(2):160-166.

27. World Health Organization. Report on the burden of endemic HAI world-wide; a systematic review of literature. Geneva: WHO; 2011.

28. Pollack, A. Rising threat of infections unfazed by antibiotics. New York Times, 2010 Feb 27.

29. Burns, K.; Foley, M.; Donlon, S. Health Protection Surveillance Centre, Point Prevalence Survey of Hospital Acquired Infections \& Antimicrobial Use in European Acute Care Hospitals: May 2012. Republic of Ireland: Critical Care Report; 2013.

30. O'Boyle CA, Henly SJ, Duckett LJ. Nurses' motivation to wash their hands: a standardized measurement approach. Appl Nurs Res 2001 Aug;14(3):136-145.

31. Tenorio AR, Badri SM, Sahgal NB, Hota B, Matushek M, Hayden MK, Trenholme GM, Weinstein RA. Effectiveness of gloves in the prevention of hand carriage of vancomycinresistant Enterococcus species by health care workers after patient care. Clin Infect Dis 2001 Mar;32(5):826-829.

32. Patterson JE, Vecchio J, Pantelick EL, Farrel P, Mazon D, Zervos MJ, Hierholzer WJ Jr. Association of contaminated gloves with transmission of Acinetobacter in an intensive care unit. Am J Med 1991 Nov;91(5):479-483. 